\title{
Avaliação do transporte de carga sedimentar no médio rio Araguaia
}

\author{
Thiago Morato de Carvalho*
}

\section{Resumo}

O estudo estima a carga de sedimentos de fundo no médio rio Araguaia, através da altura e deslocamento diário de dunas. A região estudada está localizada entre a foz do rio Crixás-Açú e a ilha do Bananal, Goiás. A velocidade média das dunas foi $7 \mathrm{~m} /$ dia, a velocidade média da água foi $1.25 \mathrm{~m} / \mathrm{s}$, correspondendo a um transporte total de 2195,60 toneladas de sedimentos de fundo/dia. Foram comparados dois métodos para verificar possíveis fontes de erro, o de Van Rijn mostrou diferença de 1414 toneladas para menos em relação ao de Struckrath, diferença de $0.06 \%$ na carga estimada entre ambos os métodos. Os resultados foram comparados com a carga de sedimento de fundo do rio Araguaia na região de Aruanã.

Palavras-chave: Rio Araguaia; Sedimentos de fundo; Métodos de Van Rijn e Struckrath.

\section{Evaluation of the sedimentary load transport of the middle Araguaia river}

\begin{abstract}
The study estimates the bed load sediment in the middle Araguaia River, through the height and dunes daily displacement. The studied region is located between the mouth of the Crixás-Açú River and the Bananal Island, Goiás. The dunes migrated at the rate of $7 \mathrm{~m} /$ day, the average water velocity was $1.25 \mathrm{~m} / \mathrm{sec}$,

Doutorando Programa Pós-Graduação em Ciências Ambientais LABOGEF/IESA - UFGO (tmorato@infonet.com.br).
\end{abstract}

Geosul, Florianópolis, v. 24, n. 47, p 147-160, jan./jun. 2009 
CARVALHO, T.M. de. Avaliação do transporte de carga sedimentar no ...

corresponding to a total transport of 2195.60 ton of bed load/day. Two methods were compared in order to verify possible sources of error, the Van Rijn one showed a difference of 1414 tons less then the Struckrath method, difference of $0,06 \%$ in the estimated bed load between both methods. Results were compared with the bed load of the Araguaia River in the region of Aruanã.

Key words: Araguaia River; Bed load sediments; Van Rijn and Struckrath methods.

\section{Introdução}

O balanço sedimentar de rios é um parâmetro importante para se entender a dinâmica dos canais, migrações de barras arenosas e formações de ilhas. Este parâmetro fluvial é a relação entre a taxa de erosão e sedimentação. Para melhor caracterizar o balanço sedimentar de um rio é preciso realizar amostragens em vários locais, de forma a permitir com que os trechos analisados possam ser comparados entre si e com estudos entre rios, como por exemplo, os relatos de sedimentos registrados no médio e baixo rio Paraná (STEVAUX et al., 1994, 1995, 2004; AMSLER, 1994, 2000, 2003; MARTINS, 2004).

Com relação ao rio Araguaia, os estudos realizados por Carvalho (2006), Latrubesse et al. (2006) e Morais (2006) contribuíram para estimar o balanço de sedimentos e avaliar a predominância da erosão ou da sedimentação na dinâmica fluvial deste rio. Estes estudos ajudaram a entender a dinâmica do Araguaia, mas outras avaliações são igualmente importantes para melhor caracterizar este rio, por exemplo, com relação aos processos erosivos e de sedimentação que ocorrem na sua planície.

Neste contexto, o presente estudo é uma caracterização do transporte de carga sedimentar de fundo no canal do médio Araguaia, entre os rios Crixás-Açú e Javaés (Ilha do Bananal). O estudo fez parte de um projeto maior sobre a morfodinâmica atual e evolução Quaternária da planície aluvial do rio Araguaia e suas 
CARVALHO, T.M. de. Avaliação do transporte de carga sedimentar no ...

implicações ambientais, desenvolvido pela Universidade Federal de Goiás, IESA.

\section{O rio Araguaia}

O rio Araguaia nasce na Serra dos Caiapós, Mato Grosso, e flui na direção norte por $2100 \mathrm{~km}$. A altitude do terreno por onde drena a bacia hidrográfica do Araguaia é 800 - 1000 metros no alto curso, prolongando-se por extensa planície fluvial, cujas cotas variam entre 200 - 500 metros. A jusante, onde se encontra com o rio Tocantins, nas proximidades de Marabá, Pará, as cotas altimétricas do Araguaia variam entre 100 - 200 metros.

$\mathrm{O}$ rio Araguaia pode ser dividido pragmaticamente em 3 trechos: alto curso, que se estende por $36.400 \mathrm{~km}^{2}$, das nascentes até Registro do Araguaia, Goiás; médio curso, que se estende por $1.600 \mathrm{~km}$, de Registro do Araguaia até Conceição do Araguaia, Pará, com uma área de $300.000 \mathrm{~km}^{2}$, onde ocorre a planície aluvial do Bananal; e baixo curso, de Conceição do Araguaia até a confluência com o rio Tocantins.

Carvalho (2004), utilizando o modelo digital de terreno, verificou a compartimentação do rio Araguaia que foi proposta por LAatrubesse e Steveaux (2002). A utilização do modelo possibilitou verificar as "quebras" (knickpoints) no perfil longitudinal do rio Araguaia, áreas chave para determinação do alto, médio e baixo rio Araguaia (Figura 2).

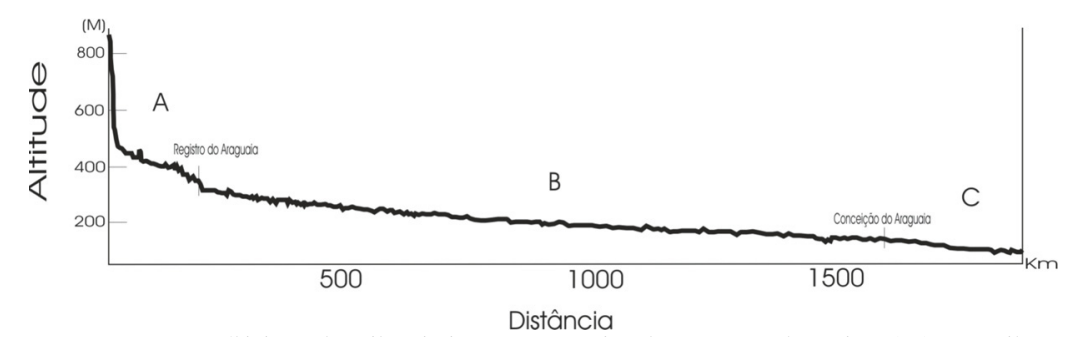

Figura 2: Perfil longitudinal dos cursos do rio Araguaia, alto (A), médio (B) e baixo (C). 
CARVALHO, T.M. de. Avaliação do transporte de carga sedimentar no ...

Souza (2002), com base no padrão de drenagem, propôs cinco divisões para a bacia do Araguaia (Figura 3). Do ponto de vista genético o Araguaia apresenta um padrão de canal misto (braided/meandriforme, BAYER 2002) com tendências ao entrelaçamento, presença de vales escavados e carga de fundo como processo sedimentológico dominante.

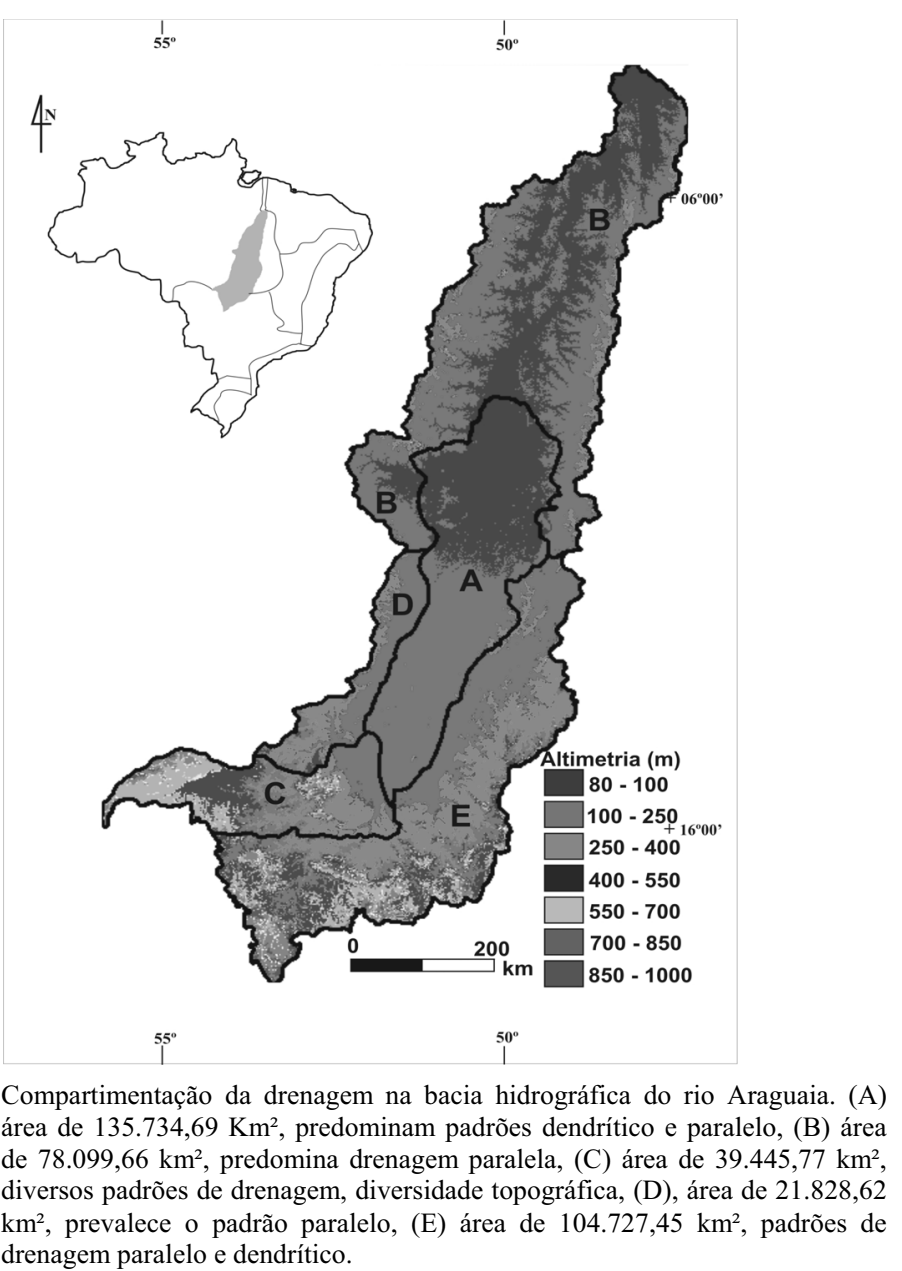


CARVALHO, T.M. de. Avaliação do transporte de carga sedimentar no ...

\section{Materiais e métodos}

Área de estudo: A região pesquisada (Figura 1) está situada no médio Araguaia. O trecho situa-se no entorno da foz do rio CrixásAçú até a bifurcação do Araguaia e Javaés (braço menor do rio Araguaia). A área referência para o local é o município de Luis Alves $\left(13^{\circ} 12^{\prime}, 50^{\circ} 34^{\prime}\right)$. Na área de estudo foram coletados e analisados: i) sedimentos em suspensão, ii) sedimentos de fundo, iii) batimetria e iv) propriedades do fluxo como velocidade e vazão.

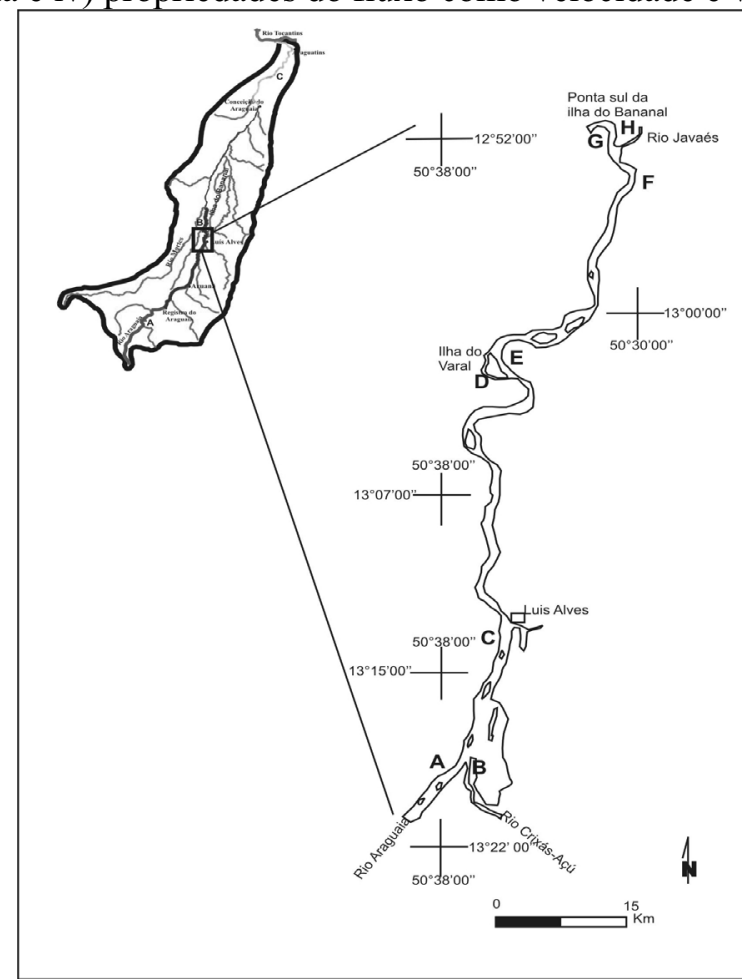

Figura 1: Área do estudo no médio rio Araguaia, entre a foz do rio Crixás-Açú e o rio Javaés (Ilha do Bananal). (A) canal principal do rio Araguaia a montante da foz do rio Crixás-Açú, (B) foz do rio Crixás-Açú, (C) canal principal do rio Araguaia a jusante do rio Crixás-Áçú (proximidades do município de Luis Alves), (D) canal do rio Araguaia defronte ilha do Varal, (E) canal do rio Araguaia $3 \mathrm{~km}$ antes da bifurcação Araguaia-Javaés, (F) braço maior do rio Araguaia $1 \mathrm{~km}$ após a bifurcação, $(\mathrm{G})$ entrada do rio Javaés (braço menor do Araguaia). 
CARVALHO, T.M. de. Avaliação do transporte de carga sedimentar no ...

Sedimentos em suspensão: As coletas foram feitas com garrafa de Van Dorn, na superfície e $60 \%$ de profundidade, ambas nas margens e no meio do canal (seção transversal). As amostras foram armazenadas em recipientes de 1 litro e mantidas em local refrigerado. Posteriormente, o material foi filtrado em bomba a vácuo e filtros de millipore $(0,45 \mu)$ para registrar o volume $(\mathrm{mg} / \mathrm{l})$ para cada amostra.

Foram utilizadas as seguintes variáveis para analisar os dados: tempo (segundos), vazão (m3/s) e média da concentração total do canal de sedimentos $(\mathrm{mg} / \mathrm{l})$. O produto destas três variáveis resulta no transporte total de sedimentos em suspensão em toneladas ao dia, estimada através da equação Qss $=\mathbf{8 6 4 0 0} \times \mathbf{Q} \times$ $\mathbf{C}=(\mathrm{g} /$ dia $)\left(\mathrm{Q}=\right.$ descarga líquida $\mathrm{em} \mathrm{m} / \mathrm{s}^{3}$ (vazão); $\mathrm{C}=$ concentração em mg/l; 86400 = segundos totais em 24 horas).

Sedimentos de fundo: As amostras de fundo foram coletadas com draga de Peterssen. As coletas foram feitas em seções transversais nas margens e no meio do canal. As amostras foram acondicionadas em sacos plásticos e transportadas ao laboratório. A determinação granulométrica foi feita através de um analisador de partículas a laser (modelo Master Size 2000). Foram estimados também os diâmetros médios dos grãos (d50).

O período analisado foi 8.4.2005 a 14.4.2005. Dois métodos foram utilizados para estimar os sedimentos de fundo: o método direto por deslocamento de dunas (STRUCKRATH, 1969) e o indireto por equação hidráulica (VAN RIJN, 1984).

Batimetria: Foi utilizada ecossonda (sensor acústico), modelo Furuno GP1650F/DF (50/200 kHz - 12/24 VDC), acoplada a um microcomputador convencional para os levantamentos batimétricos transversais e longitudinais do canal. Os perfis longitudinais foram realizados na margem esquerda, meio e margem direita. As distâncias dos perfis longitudinais foram de $1 \mathrm{~km}$, navegando no sentido do fluxo e mantendo a mesma velocidade deste. Após 6 dias da primeira medição repetiu-se o procedimento, porque é o tempo para que seja detectada de forma confiável a migração das 
CARVALHO, T.M. de. Avaliação do transporte de carga sedimentar no ...

dunas. Este procedimento foi utilizado para determinar o transporte de carga de fundo pelo método direto (STRUCKRATH, 1969).

Para determinar o transporte de carga de fundo, a amostragem dos transectos longitudinais do rio Araguaia foi feita a jusante da foz do rio Crixás-Açú, próximo à localidade de Luis Alves. Para verificar uma possível barreira arenosa na entrada do canal do rio Javaés (bifurcação Araguaia-Javaés), foi feito um transecto longitudinal partindo do rio Araguaia entrando no rio Javaés.

Os levantamentos batimétricos transversais do rio Araguaia foram realizados para plotar os pontos de amostragem dos sedimentos de fundo e em suspensão, nas respectivas profundidades onde foram coletados. Também foi feito um levantamento batimétrico transversal junto à seção dos perfis longitudinais, para verificar a morfologia do leito. Este procedimento permite separar as áreas correspondentes de cada perfil longitudinal (perfis das margens e meio do canal).

Os dados registrados pela ecosonda no computador de campo foram exportados do programa FUGAWI (utilizado para registrar os dados de batimetria) em arquivos na extensão "txt" (formato texto). Este formato permite ser importado para outros softwares, como o SURFER (utilizado para gerar os perfis batimétricos). $\mathrm{O}$ arquivo exportado diretamente do FUGAWI na extensão txt contém dados das coordenadas geográficas, distância percorrida no transecto e profundidades (batimetria).

Vazão e Velocidade do Fluxo: Para estimar estas duas variáveis foi utilizado o Acoustic Doppler Current Profiler (ADCP), também conhecido como Correntômetro Acústico de Efeito Doppler. É um instrumento que determina a velocidade do fluxo em perfis verticais. De acordo com o manual da RD Instrumentes (RDI 2001), o aparelho funciona com base no efeito Doppler, emite uma freqüência de onda sonora de $600 \mathrm{kHz}$, a uma velocidade de 1400 $1570 \mathrm{~m} / \mathrm{s}$, a qual, ao ser refletida por partículas em suspensão na água e do leito do rio, sofre alteração no comprimento de onda, modificando a freqüência. $\mathrm{O}$ sinal de retorno é usado para estimar 
CARVALHO, T.M. de. Avaliação do transporte de carga sedimentar no ...

o deslocamento relativo do alvo (partículas em suspensão e leito do rio) em relação à fonte (barco). Já que a velocidade das partículas em suspensão é a mesma do fluxo do rio, obtém-se a velocidade do fluxo.

Os dados obtidos foram processados no programa WinRiver, que armazena, processa e exibe os dados registrados pelo ADCP. Os resultados são mostrados instantaneamente pelo WinRiver, fornecendo informações das diferentes velocidades nas seções verticais do rio, vazão, vetor do fluxo, temperatura, profundidade, distância entre as margens, área da seção, velocidade relativa do barco, rumo do barco, além de dados que podem ser equacionados para estimar quantidade de sedimentos em suspensão e calcular transporte de sedimentos de fundo (MUELLER, 2002: RDI, 2001; KOSTASCHUK, et al, 2004 ).

\section{Resultados}

A Tabela 1 mostra os dados obtidos sobre os sedimentos em suspensão do rio Araguaia durante o estudo. Evidentemente no canal principal houve a maior carga de sedimentos por dia, bem como a vazão. O segundo maior volume foi no braço maior do Araguaia, na ilha do Bananal, um volume aproximadamente igual ao canal principal do rio. Isto significa que a maior parte dos sedimentos flui pelo braço maior do Araguaia na altura da ilha do Bananal. Os sedimentos carreados pelo rio Crixás-Açú contribuem marcadamente para os sedimentos carreados pelo Araguaia. 
CARVALHO, T.M. de. Avaliação do transporte de carga sedimentar no ...

Tabela 1: Rio Araguaia, curso médio, vazão, concentração média e descarga sólida de sedimentos em suspensão.

\begin{tabular}{|c|c|c|c|}
\hline Local & $\begin{array}{l}\text { Vazão } \\
\left(\mathrm{m}^{3} / \mathrm{s}\right)\end{array}$ & $\begin{array}{c}\text { Sedimentos } \\
\text { (MgL) }\end{array}$ & $\begin{array}{c}\text { Carga } \\
\text { (ton/dia) }\end{array}$ \\
\hline Canal principal & 2493,56 & 61,68 & 13288,56 \\
\hline Crixás-Açú, foz & 1347,50 & 16,20 & 1886,06 \\
\hline $\begin{array}{l}\text { Canal principal, jusante do Crixás- } \\
\text { Açú }\end{array}$ & 3636,47 & 48,20 & 15144,03 \\
\hline $\begin{array}{l}\text { Canal principal, defronte ilha do } \\
\text { Varal }\end{array}$ & 2651,88 & 45,73 & 10477,78 \\
\hline $\begin{array}{l}\text { Canal principal, } 3 \mathrm{~km} \text { antes da } \\
\text { bifurcação Araguaia-Javaés }\end{array}$ & 2743,02 & 40,03 & 9487,79 \\
\hline $\begin{array}{l}\text { Braço maior do Araguaia, } 1 \mathrm{~km} \\
\text { depois da bifurcação Araguaia- } \\
\text { Javaés }\end{array}$ & 2671,66 & 37,78 & 8720,81 \\
\hline $\begin{array}{l}\text { Entrada do rio Javaés (braço menor } \\
\text { do Araguaia) }\end{array}$ & 156,92 & 49,20 & 667,05 \\
\hline
\end{tabular}

\section{Transporte de Fundo}

i) Método direto, deslocamento de dunas (STRUCKRATH, 1969): O transporte de carga sedimentar de fundo amostrados durante 6 dias por integração das seções batimétricas longitudinais laterais e do meio do canal foi 2195,60 toneladas por dia. A velocidade das dunas no perfil batimétrico longitudinal no meio do canal foi de 7 $\mathrm{m} /$ dia. Para as seções batimétricas longitudinais laterais foram: seção próxima à margem esquerda de $3.2 \mathrm{~m} /$ dia e seção próxima à margem direita foi de $3,4 \mathrm{~m} /$ dia. A altura média das dunas (da base a crista) variou entre as seções longitudinais, sendo próximo à margem esquerda de $0.8 \mathrm{~m}$, meio do canal de $1.35 \mathrm{~m}$, e próximo à margem direita de 1.1 metros.

ii) Método indireto, equação de hidráulica (VAN RIJN, 1984): O valor calculado do transporte de carga de fundo durante 1 dia (8.4.2005) pelo método de Van Rijn, para toda a seção transversal do canal, foi 2.194,190 toneladas por dia. Este valor corresponde ao transporte de sedimentos de fundo para uma velocidade média 
CARVALHO, T.M. de. Avaliação do transporte de carga sedimentar no ...

do fluxo de $1,25 \mathrm{~m} / \mathrm{s}$, profundidade média de $7,3 \mathrm{~m}$ e um D50 de $0.433 \mathrm{~mm}$.

\section{Discussão}

A descarga do fluxo de água no trecho estudado apresentou pequenas variações, em torno de 2.500 a $3.000 \mathrm{~m}^{3} / \mathrm{s}$. O trecho que variou mais foi na foz do rio Crixás-Açú, devido ao incremento do volume de água, e variações ao longo do trecho até a bifurcação do rio Araguaia e o rio Javaés. Nesta bifurcação o fluxo se divide nos dois canais. Este comportamento da variação da vazão em diferentes pontos do Araguaia foi estudado através de análises de hidrogramas por Aquino (2002). Ela relata que o Araguaia no seu médio curso, desde Aruanã até a Fazenda Telésforo (a jusante da bifurcação Araguaia-Javaés), tem uma tendência a perder água (cerca de $30 \%$ de seu volume total), que em parte deriva para a planície fluvial e em outra parte para o rio Javaés. Este fato também foi observado no presente estudo, os valores de vazão decrescem da montante para jusante, indicando perda de volume de água.

O transporte de sedimentos em suspensão no trecho entre a montante do rio Crixás-Açú e a bifurcação do Araguaia-Javaés apresentou variações na concentração nas seções transversais, entre as margens e o meio do canal. A descarga sólida (sedimentos em suspensão) variou ao longo do trecho, conforme a variação da descarga líquida já citada anteriormente. O total de descarga sólida em suspensão no trecho próximo a cidade de Luis Alves foi 15.144,035 toneladas por dia.

O valor da carga de sedimentos em suspensão obtido nesta pesquisa é inferior ao valor médio estimado por Carvalho (1994), que utilizou os mesmos métodos empregados no presente estudo para estimar a concentração de sedimentos em suspensão. $\mathrm{O}$ valor médio diário foi 46.791 toneladas para a estação de Luis Alves, próximo ao ponto deste estudo. Esta diferença de menos da metade do valor médio anual estimado por Carvalho (1994) é explicada pelo fato de o rio Araguaia apresentar grandes pulsos de 
CARVALHO, T.M. de. Avaliação do transporte de carga sedimentar no ...

concentração de sedimentos em suspensão em diferentes épocas do ano. O presente estudo foi feito no final da estação chuvosa, época durante a qual os valores de concentração de sedimentos em suspensão estão abaixo da média nas proximidades de Luis Alves.

De acordo com o método direto de Struckrath (1969) o transporte de carga de fundo no trecho defronte à cidade de Luis Alves (jusante do Crixás-Açú) foi 2.195,604 toneladas por dia. O método indireto de Van Rijn foi 1.414 toneladas a menos, uma diferença de $0.06 \%$. O resultado do presente estudo avaliou os sedimentos durante uma semana, para 1 ano teríamos algo em torno de 801.395,460 ton/ano. O transporte de carga de sedimentos de fundo em Aruanã é 487.441 ton/ano. Comparado com os dados de Luis Alves, a diferença é 313.954 ton/ano. Isto indica que entre estes dois trechos há um incremento de aproximadamente 313.954 ton/anual de carga de fundo no rio Araguaia, a partir de Aruanã. Com relação à morfologia de fundo, não ocorre variações marcantes entre os dois trechos, que apresentam médias aproximadas da altura das dunas e os mesmos deslocamentos diários.

\section{Agradecimentos}

A equipe do LABOGEF e aos professores Laerte Guimarães Ferreira, Leandro Gonçalves, Celso Morato de Carvalho e Selma Simões de Castro pelas sugestões.

\section{Referências bibliográficas}

AMSLER, M.; DRAGO, E.C. 2003. A review of the suspended sediment budget at the confluence of the Paraná and Paraguay Rivers. Hydrology Processes, v.32. 32-43p.

AMSLER, M.; GAUDI, H.E. 1994. La susperposicíon de dunas y el transporte de la carga de fondo en el río Paraná. In: Congresso Nacional del Agua. La Plata. v.3. 1-10p. 
CARVALHO, T.M. de. Avaliação do transporte de carga sedimentar no ...

AMSLER, M.L.; PRENDES, H.H. 2000. Transporte de sedimentos y processos fluviales associados. In: El Río Paraná em su trama médio, Contribuicion al conocimiento y practicas ingenieriles em um gran rio de llanura, Tomo 1. centro de publicaciones, UNL, Santa Fé, Argnetina. Cap.5. 247-253p.

AQUINO, S.S. 2002. Regime Hidrológico e Comportamento Morfo hidráulico do rio Araguaia. Dissertação (Mestrado em Geografia) Universidade Estadual de Maringá, Maringá, PR. 113p.

BAYER, M. 2002. Diagnóstico dos processos de erosão/assoreamento na planície aluvial do rio Araguaia: entre Barra do Garças e Cocalinho. P.138. Dissertação (Mestrado em Geografia) Instituto de Estudos Sócio-Ambientais, Universidade Federal de Goiás, Goiânia.

CARVALHO, T.M. 2004. Aplicação de Modelos Digitais do Terreno (MDT) em Análises Macrogeomorfológicas: o Caso da Bacia Hidrográfica do rio Araguaia. Revista Brasileira de Geomorfologia, N1, 85-93p.

CARVALHO, T.M. 2006. Transporte de Carga Sedimentar no Médio rio Araguaia entre os rios Crixás-açú e Javaés. P.110. Dissertação (Mestrado em Geografia) Instituto de Estudos SócioAmbientais, Universidade Federal de Goiás, Goiânia.

CARVALHO, N.O. 1994. Hidrossedimentologia Prática. Editora da CPRM/Rio de Janeiro. 372p.

KOSTASCHUK, R.A.; VILLARD, P.V.; BEST, J.L. 2004. Measuring flow velocity and sediment transport with an acoustic Doppler current profiler. Geomorphology, v.68.25-37p.

LATRUBESSE, E.M.; AMSLER, M.; MORAIS,R.P. 2006. The geomorphologic response of a large pristine alluvial river tremendous deforestation in the South American tropics: the Araguaia case. Earth Surface Process and Landforms (no prelo). 
CARVALHO, T.M. de. Avaliação do transporte de carga sedimentar no ...

LATRUBESSE, E.M.; STEVAUX, J.C. 2002. Geomorphology and environmental aspects of the Araguaia fluvial basin, Brazil.Z.Geomorph.N.F. Berlin, Suppl.-Bd.129, 109-127p.

LEOPOLD, L.; WOLMAN, M.G.; MILLER, J.P. 1964. Fluvial process in Geomorphology. San Francisco: Editora H.W. Freeman. 201p.

MORAIS, R.P. 2006. A Planície Aluvial do Médio rio Araguaia: Processos Geomorfológicos e Suas Implicações Ambientais. Tese (Doutorado em Ciências Ambientais) Instituto de Ciências Ambientais, Universidade Federal de Goiás, Goiânia. 135p.

MUELLER, D.S. 2002. Field assessment of acoustic-Doppler based discharge measurements. Proceedings of Hydraulic Measurements and Experimental Methods. American Society of Civil Engineers. 95-102p.

RD Instruments, Inc. 2001. WinRiver user's guide-USGS version: San Diego, Calif., RD.

STEVAUX, J.C. 1994. The upper Paraná river (Brazil): Geomorphology, sedimentology and paleoclimatic. Quaternary International, v.21, 1430161p.

STEVAUX, J.C.; FILHO, E.E.; MARTINS, D.P. 2004. Chracteristics and dynamics of the Paraná river channel bedform: na approuch to study the influences of the Porto Primavera Dam, Sand mining, and channel construction. In: Structure and function of the Paraná river and its floodplain. 15-18p.

STEVAUX, J.C.; TAKEDA, A.M. MORAIS, M. 1995. Dinâmica sedimentar no canal do rio Paraná. In: $6^{\circ}$. Simpósio Sul-Brasileiro de Geologia, Porto Alegre, Boletim de Resumos Expandidos, v.1. 284-297p. 
CARVALHO, T.M. de. Avaliação do transporte de carga sedimentar no ...

STEVAUX, J. C.; Souza Filho, Edvard E.; Martins, D. P., 2003 Característica e Dinâmica as Formas de Leito do Rio Paraná: Considerações Sobre a Barragem de Porto Primavera, a Extração de Areia do Canal e a Implantação da Hidrovia do Rio Paraná. UEM. Maringá. Field Conference, Guide Amazon, Pg. 27-30.

STRUCKRATH, T. 1969. Movimento de las ondulaciones del lecho de río Paraná. Mitteilungen das Frazius Institue. Editora da Universitat Hannover, v.1. 20p.

VAN RIJN, L.C. 1984. Sediment transport, PartI: Bed Load Transport. Journal of Hydraulic Engineering. ASCE. V.110. 23$31 \mathrm{p}$.

Recebido em setembro de 2007 Aceito em junho de 2008 